\title{
Les tropomyosines
}

Les tropomyosines sont des protéines ubiquitaires, très conservées au cours de l'évolution, associées à l'actine polymérisée. Elles contribuent à la régulation de la contraction du muscle strié. Dans les autres tissus, elles participent au cytosquelette et à la motilité cellulaire. La diversité des tropomyosines est due à l'existence de plusieurs gènes, mais aussi à la synthèse d'isoformes multiples à partir d'un même gène, par l'utilisation de promoteurs multiples et d'exons alternatifs, sous un contrôle spécifique de chaque tissu et de différentes étapes du développement de ce tissu.

\section{Marguerite Lemonnier Domenico Libri Vincent Mouly Marc Y. Fiszman}

\section{ADRESSE}

M. Lemonnier : docteur es sciences, chargée de recherche Inserm. D. Libri : docteur es sciences. V. Mouly : docteur es sciences, chargé de recherche Cnrs. M.Y. Fiszman : docteur es sciences, directeur de recherche Cnrs. Unité de biochimie, Cnrs UA 1148, Institut Pasteur, 25-28, rue du Docteur-Roux, 75724 Paris Cedex 15, France.

$\mathrm{m} / \mathrm{s} n^{\circ} 7$ vol. 6 , septembre 90 solées à l'origine à partir des muscles squelettiques de vertébrés [1], les tropomyosines sont représentées chez tous les eucaryotes, de la levure à l'homme. Les nombreuses isoformes identifiées dans toutes les espèces partagent des caractéristiques structurales fondamentales : conservation de la séquence peptidique, structure en double hélice de la protéine native et association avec l'actine polymérisée (actine F). Pourtant, ces isoformes de la tropomyosine assurent des fonctions différentes : dans les muscles, elles participent à la régulation de la contraction et dans les tissus non musculaires, elles sont impliquées dans la motilité cellulaire.

\section{Tropomyosines du tissu musculaire}

Les premières à avoir été isolées, ces tropomyosines sont les mieux con- nues d'un point de vue structural et fonctionnel. Avec l' $\alpha$-actine et le complexe des troponines (troponine $\mathrm{T}$, troponine $\mathrm{C}$ et troponine $\mathrm{I}$ ), elles sont des constituants du filament fin du sarcomère qui règle le couplage excitation - contraction dans les muscles striés.

En bref, le sarcomère est le motif élémentaire dont la répétition constitue les myofibrilles. Le sarcomère est formé par l'assemblage de protéines organisées en filaments épais et en filaments fins susceptibles de glisser l'un sur l'autre : ces filaments épais et fins sont rattachés à des structures ayant un aspect de bandes en microscopie électronique, appelées respectivement bandes $M$ et $Z$. Le filament épais est constitué de myosine (chaînes lourdes et légères). Le filament fin est constitué d'actine polymérisée, de tropomyosine polymérisée et de troponines (troponines $\mathrm{T}$, I et $\mathrm{C}$ ). 


\section{RÉFÉRENCES}

1. Bailey K. Tropomyosin : a new asymetric protein component of the muscle fibril. Biochem J 1948 ; 43 : 271-2.

2. McLachlan AD, Stewart M. Tropomyosin coiled-coil interactions : evidence for an unstaggered structure. J Mol Biol 1975 ; 98 : 293-304.

3. White SP, Cohen C, Phillips Jr GN Structure of co-crystals of tropomyosin and troponin. Nature $1987 ; 325$ : 826-8.

4. Schachat FH, Diamond MS, Brandt PW. Effect of different troponin Ttropomyosin combinations on thin filament activation. J Mol Biol 1987; 198 : 551-4.

5. Leger J, Bouveret P, Schwartz K, Swynghedauw B. A comparative study of skeletal and cardiac tropomyosins. Pflugers Arch 1976 ; 362 : 271-7.

6. Montarras D, Fiszman MY, Gros F. Characterization of the tropomyosins present in various chick embryo muscle types and muscle cells differentiated in vitro. J Biol Chem 1981; 256 : 4081-6.

7. Giometti CS, Anderson NL. Tropomyosin heterogeneity in human cells. $J$ Biol Chem 1984; 259: 14113-20.

8. Hendricks M, Weintraub H. Multiple tropomyosin polypeptides in chicken embryo fibroblasts : differential repression of transcription by Rous Sarcoma virus transformation. Mol Cell Biol 1984 ; 4 : 1823-33.

9. Matsumura F, Yamashiro-Matsumura S. Purification and characterization of multiple isoforms of tropomyosin from rat cultured cells. J Biol Chem 1985; 260 : 13851-9.

10. Liu H, Bretscher A. Disruption of the single tropomyosin gene in yeast results in the disappearance of actin cables from the cytoskeleton. Cell 1989; $57: 233-42$.

11. Hegman TE, Lin JL-C, Lin JJ-C. Probing the role of nonmuscle tropomyosin isoforms in intracellular granule movement by microinjection antibodies. J Cell Biol 1989 ; 109 : 1141-52.

12. Broschat $\mathrm{KO}$, Burgess DR. Low $\mathrm{Mr}$ tropomyosin isoforms from chicken brain and intestinal epithelium have distinct actinbinding properties. J Biol Chem 1986 ; 261 : 13350-9.

13. Hanke PD, Storti RV. The Drosophila melanogaster tropomyosin II gene produces multiple proteins by use of alternative tissue-specific promoters and alternate splicing. Mol Cell Biol 1988; 8 : 3591-602.

14. Libri D, Lemonnier M, Meinnel $T$, Fiszman MY. A single gene codes for the $\alpha$ subunit of smooth and skeletal muscle tropomyosin in the chicken. J Biol Chem 1989 ; 264: 2935-44.

15. Lindquester GJ, Flach JE, Fleenor DE, Hickman KH, Devlin RB. Avian tropomyosin gene expression. Nucleic Acids Research 1989 ; 17 : 2099-117.
Dans le sarcomère, les tropomyosines sont sous forme d'un filament provenant de la polymérisation tête-àqueue d'un dimère assemblé à partir de deux sous-unités, identiques ou différentes, de 284 acides aminés chacune. La double hélice superenroulée de tropomyosine polymérisée occupe le sillon de l'hélice de l' $\alpha$ actine polymérisée (actine F). Chaque dimère de tropomyosine est en contact avec sept molécules d'actine (figure 1).

L'analyse des séquences peptidiques des sous-unités connues de tropomyosine de muscle strié fait apparaître un motif répété de 7 acides aminés représentant environ deux tours d'hélice (3,62 aminoacides par tour d'hélice) (figure 2). Le premier acide aminé (a) du motif et le $4^{\mathrm{e}}$ (d) sont toujours des acides aminés hydrophobes : ils interagissent avec les acides aminés correspondants (d') et (a') de la deuxième sous-unité (figure 2) [2]. Ces interactions sont à l'origine de la structure en double hélice. Cette double hélice est également stabilisée par des interactions salines entre les acides aminés chargés qui se trouvent le plus fréquemment en position 5 (e) et 7 (g) du motif répété. Les autres acides aminés ne sont pas ou peu impliqués dans la formation de la double hélice et sont, de ce fait, disponibles pour interagir avec d'autres molécules du filament fin comme l'actine ou les troponines.

Comment les tropomyosines contribuent-elles à la régulation de la contraction musculaire? L'analyse de diffraction des rayons $\mathrm{X}$ par des cristaux de la protéine, seule ou associée in vitro avec d'autres protéines du filament fin, a montré que la tropomyosine pourrait adopter plusieurs états transconformationnels à l'intérieur du sillon d'actine :

- pour des concentrations en calcium faibles (inférieures à $10^{-8} \mathrm{M}$ ), la tropomyosine est associée au complexe des troponines, et en particulier à la troponine $\mathrm{T}$ par deux domaines. Le premier domaine d'interaction se trouve dans la zone de recouvrement de deux sous-unités successives, recouvrement qui assure la polymérisation de la tropomyosine : cette interaction tropomyosine - troponine $\mathrm{T}$ ne dépendrait pas de la concentration en calcium. Le deuxième domaine d'interaction est trouvé dans la région de la tropomyosine proche de la cystéine en position 190 : cette interaction dépendrait de la concentration en calcium et serait forte en l'absence de saturation de la troponine- $\mathrm{C}$ par le calcium [3]. Dans cette configuration, la tropomyosine serait maintenue au bord du sillon de l'actine polymérisée (figure 3a, p. 648) : c'est l'état " inactif";

- lorsque la concentration du calcium intracellulaire augmente, la fixation du calcium sur la troponine C (TN-C) entraînerait un changement de conformation du complexe des troponines. Le contact tropomyosine - troponine $\mathrm{T}$ se limiterait à la seule interaction indépendante de la concentration en calcium, dans le domaine de recouvrement de deux dimères successifs de tropomyosine [3]. La tropomyosine se déplacerait alors vers l'intérieur du sillon de l'hélice de l' $\alpha$-actine polymérisée (état " potentialisé ") (figure 3b). Cette nouvelle conformation du filament fin permet l'interaction des têtes de myosine $\mathrm{S} 1$ avec l'actine (figure 3c), cette interaction renforçant également, par un effet coopératif, le déplacement de la tropomyosine dans le sillon de l'actine. C'est dans cet état "actif" qu'intervient l'hydrolyse de l'ATP. Des études ultérieures devraient permettre d'établir définitivement les propriétés de la tropomyosine dans le filament fin reconstitué et dans la fibre musculaire.

Les tropomyosines natives du muscle squelettique sont constituées de deux sous-unités différentes, de 284 acides aminés, classées $\alpha$ ou $\beta$ en fonction de leur mobilité électrophorétique et de leur carte peptidique, assemblées sous forme d'homodimère ou d'hétérodimère. Dans les fibres classées "lentes " ou " rapides" (en fonction de l'activité ATPasique du complexe actomyosine qu'elles contiennent) sont exprimées des sous-unités différentes de tropomyosine : $\alpha \mathrm{f}$ et $\beta$ dans toutes les fibres musculaires rapides, $\alpha$ s et $\beta$ dans les fibres lentes. Par ailleurs, dans des fibres rapides différentes, des complexes spécifiques sont formés entre les dimères de tropomyosine et des isoformes de la troponine $\mathrm{T}: \alpha \mathrm{f} 2 / \mathrm{TN}-\mathrm{T}-3 \mathrm{f}$ et $\alpha \mathrm{f} \beta / \mathrm{TN}-$ T-1f. Il a été montré que, placées en 


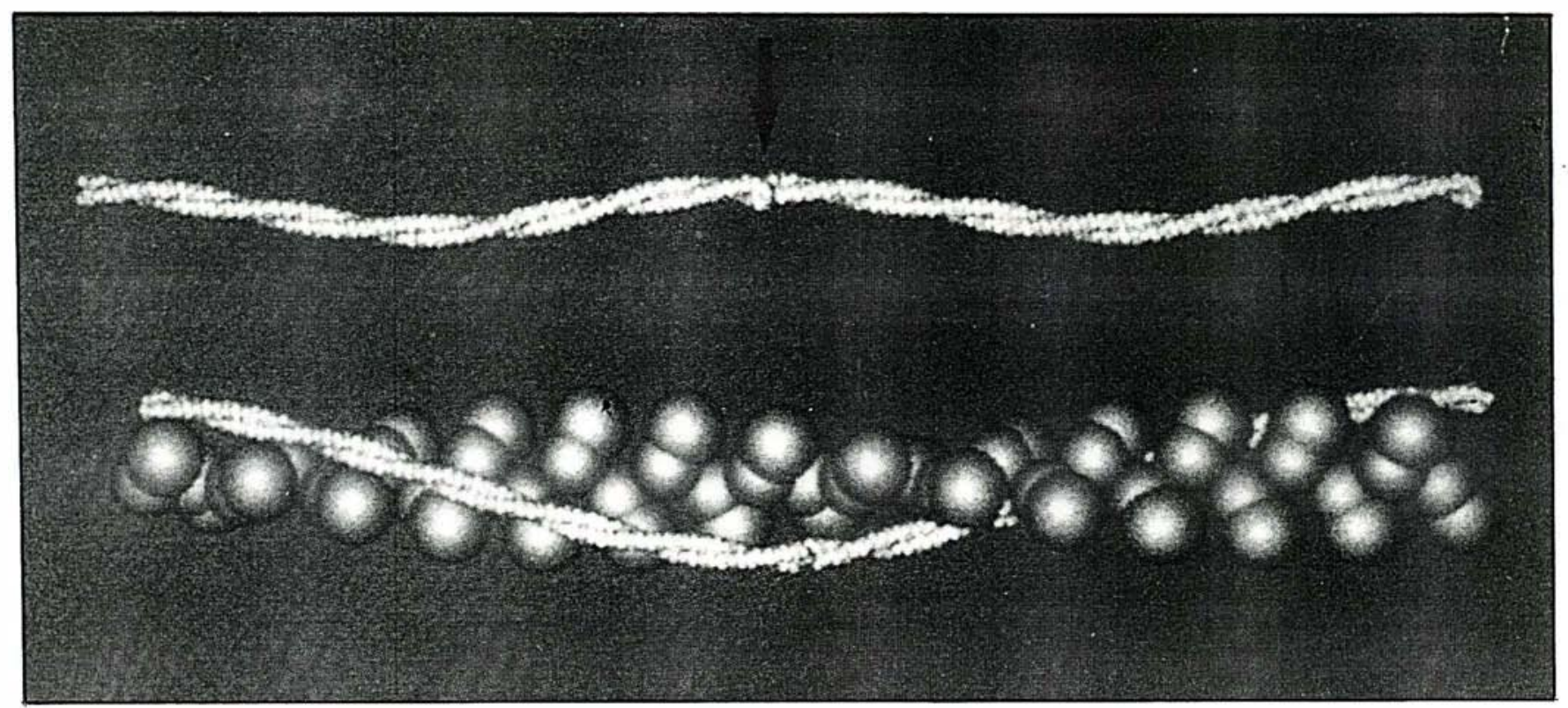

Figure 1. Représentation de la structure enroulée de la tropomyosine polymérisée isolée et dans le sillon de I'actine [29]. Un dimère de tropomyosine (composé de deux sous-unités identiques ou différentes) est en contact avec 7 monomères d'actine par demi-tour de l'hélice d'actine. La polymérisation des dimères de tropomyosine se fait par recouvrement (voir la flèche) des extrémités $C$ - et $N$ - terminales de deux dimères successifs. L'aptitude à polymériser d'un dimère est fonction de la nature des séquences $\mathrm{N}$ - et $\mathrm{C}$ - terminales des sous-unités qui le constituent.

conditions isométriques et pour une même concentration en calcium, ces différentes fibres produisent des tensions différentes en fonction des complexes tropomyosine-troponine $\mathrm{T}$ qu'elles contiennent [4]. Ce résultat suggère, entre autres, que les sousunités $\alpha$ et $\beta$ de la tropomyosine ont des propriétés fonctionnelles différentes.

En ce qui concerne les isoformes de la tropomyosine exprimées dans le muscle cardiaque des mammifères, elles sont identiques à celles du muscle squelettique rapide : $\alpha$ f et $\beta$ chez les grands mammifères (homme, mouton), $\alpha$ f chez les petits mammifères (rat, souris) [5]. En revanche, chez les oiseaux, il existe une sousunité de tropomyosine spécifique du muscle cardiaque $\alpha c$ [6].

Des sous-unités spécifiques des muscles lisses $\alpha$ sm et $\beta$ sm ont par ailleurs été caractérisées chez tous les vertébrés. De même que les isoformes du tissu musculaire, elles existent à l'état polymérisé, et les différences observées dans la polymérisation des dimères sont essentiellement liées à des différences dans la séquence peptidique C-terminale impliquée dans le recouvrement de deux dimères suc$m / s n^{\circ} 7$ vol. 6 , septembre 90

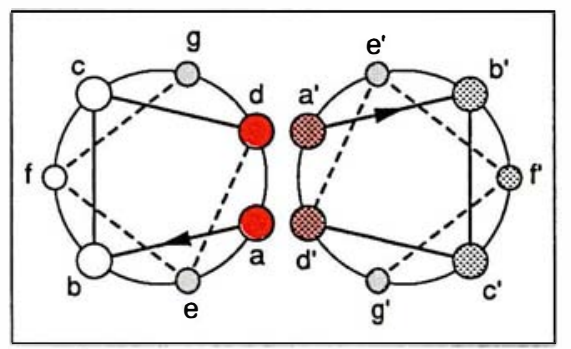

Figure 2. Interaction des sousunités dans la tropomyosine native d'après [30]. La tropomyosine native est assemblée à partir de deux sousunités identiques ou différentes. Ces sous-unités sont constituées d'un motif répété de 7 acides aminés : a $b-c-d-e-f-g$, dans lequel la répartition des acides aminés polaires et non polaires conduit à la prédiction d'une structure hélicoïdale. Les acides aminés a et $d$ sont non polaires et les acides aminés $e$ et $g$ sont le plus souvent polaires. Les deux sous-unités de la tropomyosine native s'assemblent par interactions hydrophobes entre les résidus $a, d$ et $a^{\prime}, d^{\prime}$. Le dimère est stabilisé par les interactions salines entre les chaînes latérales des acides aminés $e, g$ et $e^{\prime}, g^{\prime}$, dans une configuration parallèle (N-C/N-C). La disposition du motif de base de deux sousunités est montrée sous forme d'une projection sur un plan de coupe transversal de la tropomyosine native. cessifs. En l'absence de troponines dans les muscles lisses, le rôle des tropomyosines serait de contribuer à la régulation de la réponse du filament fin au calcium en relation avec d'autres protéines liant le calcium (caldesmone...).

\section{Tropomyosines non musculaires}

Les tropomyosines du tissu non musculaire (de 3 à 5 sous-unités différentes de 247, 248 ou 284 aminoacides) sont moins bien connues [7-9]. Par rapport aux isoformes musculaires, leur capacité à former des polymères est moins grande, voire nulle, et elles s'associent à l'actine polymérisée exclusivement sous forme d'homodimères. Des études immunocytochimiques ont suggéré qu'une de leurs fonctions serait de stabiliser les microfilaments d'actine du cytosquelette des cellules. Ces microfilaments sont impliqués dans le cytosquelette et dans la motilité cellulaire, c'est-àdire dans l'ensemble des mouvements cellulaires : migrations cellulaires au cours de la morphogenèse et tous les mouvements intracellulaires. La stabilisation des microfilaments par les 


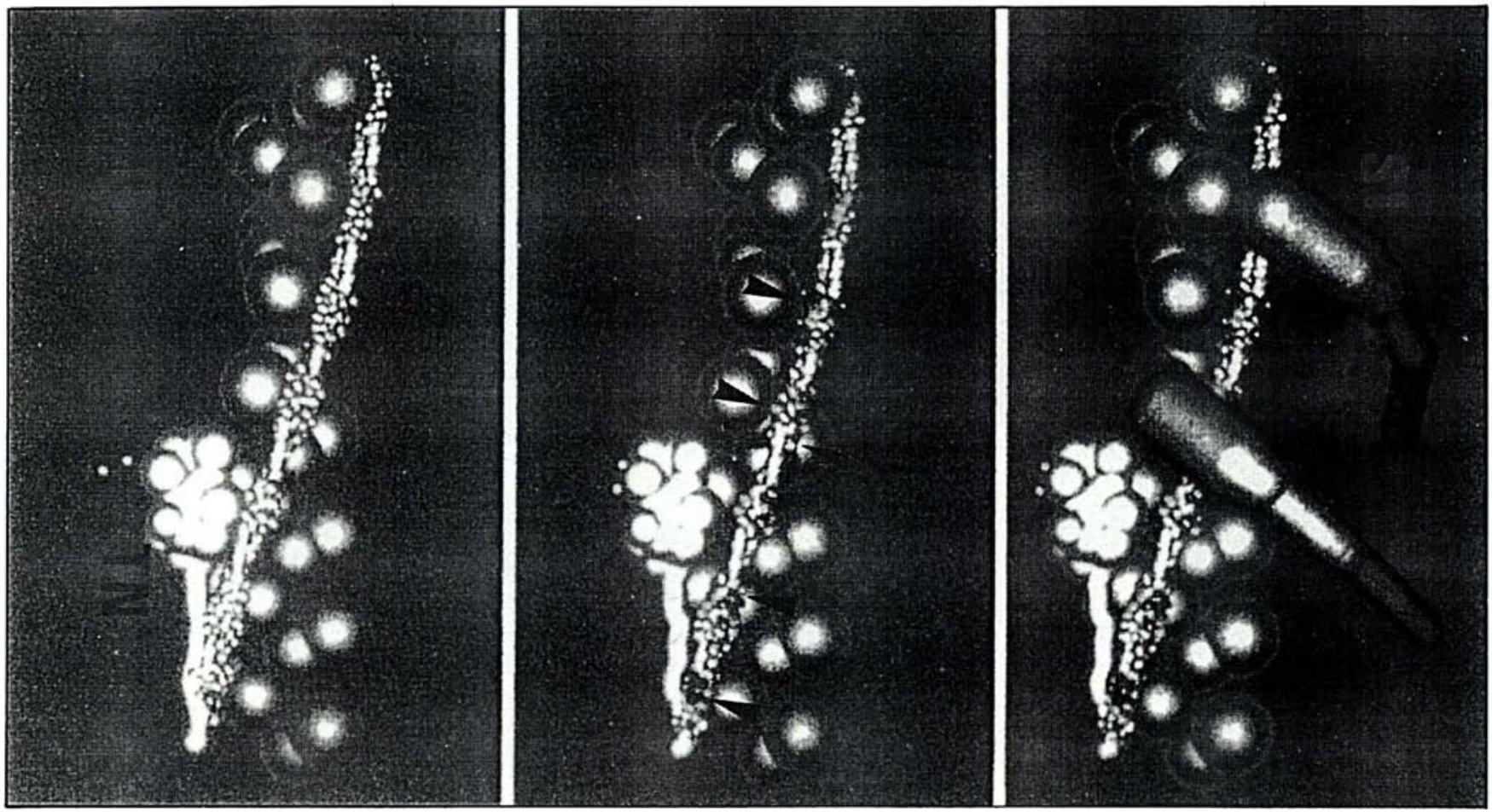

Figure 3. États transconformationnels de la tropomyosine polymérisée dans le filament fin du sarcomère [29]. a : dans l'état " inactif ", le complexe des troponines (TN) maintient le filament de tropomyosine (TM) sur le bord externe de l'hélice d'actine $(A)$ par une interaction troponine $T$ tropomyosine, dans deux régions : la région de recouvrement de deux dimères successifs et la région de la cystéine 190; b : dans l'état "potentialisé ", le complexe des troponines a lié le calcium et relâché son action sur la tropomyosine dans la région de la cystéine 190. La tropomyosine occupe alors une nouvelle place dans le sillon de l'actine polymérisée (voir les flèches). Cela permet le début de l'interaction des têtes de myosine avec l'actine. $c$ : dans l'état "actif ", les interactions entre l'actine et la tropomyosine se sont renforcées de façon coopérative et sont plus régulières. Dans cette conformation, un grand nombre de têtes de myosine (S1) sont liées à l'actine et l'hydrolyse de l'ATP peut intervenir.

tropomyosines serait due à l'inhibition de l'action de facteurs détruisant les microfilaments (gelsoline ou le facteur de dépolymérisation de l'actine). Ce rôle dans la stabilisation des filaments d'actine vient d'être démontré chez la levure, grâce à l'utilisation de mutants défectifs pour la tropomyosine [10]. Par ailleurs, la microinjection d'anticorps dirigés contre une isoforme spécifique des tropomyosines non musculaires, dans des cultures de fibroblastes, est suivie de la perte temporaire de mobilité des particules subcellulaires ; dans cette expérience, cependant, il n'y a pas de modifications importantes du cytosquelette et les rôles des isoformes non musculaires de la tropomyosine restent à préciser [11].

Les différentes tropomyosines non musculaires, placées dans des conditions physiologiques de $\mathrm{pH}$, de force ionique et de concentration en magnésium, ont des affinités pour l'actine très variables (dans un rapport de 1 à 10) en relation avec les exigences particulières de chaque tissu : isoformes à forte affinité pour l'épithélium intestinal dont le cytosquelette est très organisé, isoformes à faible affinité dans un environnement nécessitant une plus grande plasticité, les cônes de croissance des neurones par exemple [12].

\section{Organisation et origine des gènes de la tropomyosine}

La diversité des tropomyosines résulte de la combinaison de plusieurs éléments. Tout d'abord, à l'exception des levures, les gènes de tropomyosine sont multiples : deux chez la drosophile, au moins cinq chez les oiseaux et quatre chez les mammifères. De plus, l'utilisation à l'intérieur d'un même gène d'exons spécifiques alternatifs et d'extrémités 5 ' et 3' traduites distinctes (figure 4) peut engendrer une grande diversité de protéines à partir d'un locus unique. Il faut noter que l'utilisation d'extrémités $\mathrm{N}$-terminales distinctes conduit à des protéines de longueur différente (247-248 acides aminés au lieu de 284-285 acides aminés).

Comme de très nombreux gènes eucaryotes, les gènes de tropomyosine sont découpés en exons (séquences codantes) et introns, à l'exception du gène de tropomyosine de levure. Dans le cas des tropomyosines, chaque exon représente approximativement 21 ou 42 acides aminés, soit un multiple du motif de base de 7 acides aminés qui constitue la protéine (figure 2). Cela suggère que les gènes 
se sont formés par propagation d'un exon " primordial ". La similitude du découpage des séquences codantes des gènes de tropomyosine de drosophile et de mammifères a conduit à postuler l'existence d'au moins un gène ancestral établi avant la séparation des vertébrés et des invertébrés (environ 500 millions d'années). Au cours de l'évolution, ce gène aurait donné chez la drosophile le gène très morcelé TmII [13] alors que chez les vertébrés, ce gène aurait pu produire, par duplication génique, quatre ou cinq gènes évoluant alors séparément chez les oiseaux $[14,15]$ et les mammifères [16-18] (figure 5 p. 651). Sur ce point, l'isolement d'ADNc et des gènes correspondants de tropomyosines d'autres espèces chez les nématodes, les poissons ou les batraciens - apportera des indications intéressantes.

Le deuxième élément qui contribue à la diversité des tropomyosines est la duplication d'exons utilisés alternativement, à l'intérieur d'un gène unique (figure 4). De ce point de vue, les gènes de tropomyosine de vertébrés présentent entre eux une parenté certaine avec l'utilisation des mêmes couples d'exons alternatifs : $2 \mathrm{a}$ et $2 \mathrm{~b}$ (gènes $\alpha \mathrm{fTM}$ ), $6 \mathrm{a}$ et $6 \mathrm{~b}, 9 \mathrm{a}$ et $9 \mathrm{~b}$ (gènes $\alpha \mathrm{f}, \beta$ et $\alpha \mathrm{s} \mathrm{TM}$ ). Chez Drosophila melanogaster (invertébré), les exons dupliqués peuvent correspondre à des régions différentes de la protéine (exons $5 \mathrm{a}$ et $\mathrm{b}, 6 \mathrm{a}$ et $\mathrm{b}$, mais aussi 9a, b, c et d).

Dans une espèce donnée, les membres d'une paire d'exons alternatifs ont divergé, alors que l'un d'entre eux peut être très conservé par rapport à l'exon équivalent dans une autre espèce : à titre d'exemple, les séquences nucléotidiques des exons $6 \mathrm{a}$ et $6 \mathrm{~b}$ du gène $\alpha \mathrm{f} T \mathrm{M}$ de poulet n'ont que $50 \%$ de conservation alors que les séquences nucléotidiques des exons $6 \mathrm{~b}$ du gène $\alpha \mathrm{f} \Gamma \mathrm{M}$ du poulet de l'homme sont conservées à $92 \%$. $\mathrm{La}$ conservation de la séquence nucléotidique et donc peptidique de cet exon $6 b$ pourrait signer une fonction particulière. De fait, dans le sarcomère, cet exon correspond à une région de la protéine impliquée dans la liaison avec la troponine $\mathrm{T}$ (au voisinage de la cystéine 190). D'autres régions des tropomyosines sont conservées, en particulier la $\mathrm{m} / \mathrm{s} n^{\circ} 7$ vol. 6 , septembre 90

\section{Panneau a}

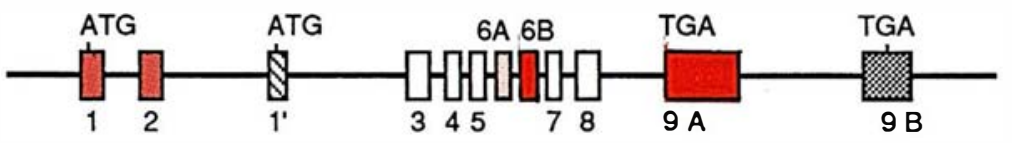

A $\mathrm{nm} \beta \mathrm{TM}$

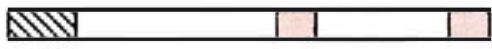

B $\beta$ TM

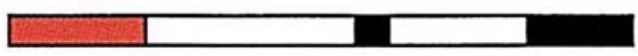

sm $\beta$ TM

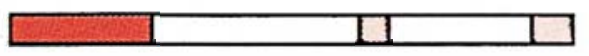

\section{Panneau b}

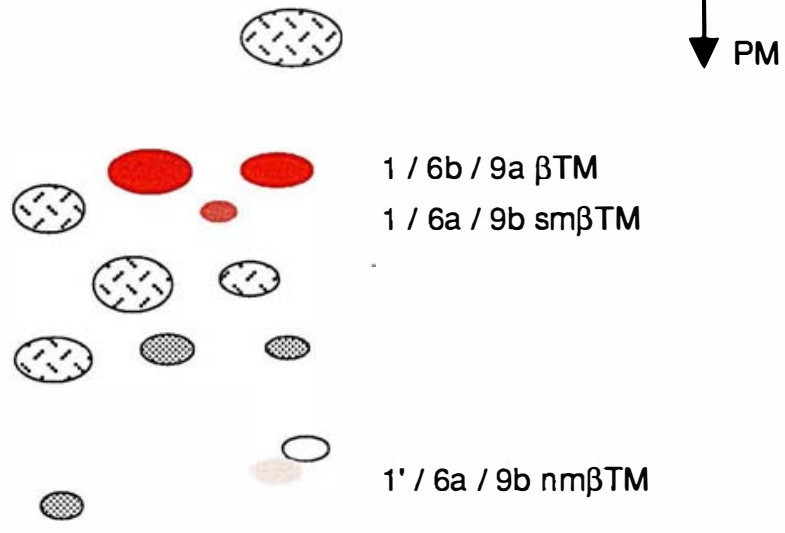

Figure 4. Représentation schématique du gène $\beta$-tropomyosine, des messagers matures et des protéines qui sont synthétisés à partir de ce locus chez les oiseaux. Panneau a : dans les tissus non musculaires, seul le transcrit mature $A$ est produit ; il code pour la protéine nmBTM 1247 acides aminés). Lors de la différenciation du tissu musculaire strié, l'initiation de la transcription est reportée de l'exon $1^{\prime}$ vers l'exon 1, la transcription est activée et l'épissage du transcrit primaire conduit au transcrit mature $B$ codant pour la protéine $\beta$ TM de 284 acides aminés. Dans le muscle lisse, le transcrit mature $C$ produit code également pour une protéine de 284 acides aminés sm $\beta$ TM, différente de celle accumulée dans le muscle squelettique smßTM. Panneau $b$ : représentation de la séparation de ces différentes isoformes par électrophorèse en gels de polyacrylamide bidimensionnels. L'isoforme $\beta$ TM existe sous forme phosphorylée ou non phosphorylée et peut apparaître en deux taches sur le gel. La nature des exons alternatifs trouvés dans les messagers codant pour ces isoformes est donnée à droite du schéma. Les taches non identifiées sur la figure correspondent à des isoformes de tropomyosines produites à partir du gène afTM (taches hachurées) ou du gène $\alpha s T M$ (taches quadrillées). 


\section{RÉFÉRENCES}

16. Helfman DM, Cheley S, Kuismanen E, Finn LA, Yamawaki-Kataoka Y. Non muscle and muscle tropomyosin isoforms are expressed from a single gene by alternative RNA splicing and polyadenylation. Mol Cell Biol 1987 ; 6 : 3582-95

17. Wieczorek DF, Smith CWJ, NadalGinard B. The rat $\alpha$ tropomyosin gene generates a minimum of six different mRNAs coding for striated, smooth and nonmuscle isof orms by alternative splicing. Mol Cell Biol 1988 ; 8 : 679-94.

18. Clayton L, Reinach FC, Chumbley GM, MacLeod AR. Organization of the hTMnm gene : Implication for the evolution of muscle and non-muscle tropomyosins. J Mol Biol 1988 ; 201 : 507-15.

19. Minty A, Kedes L. Upstream regions of the human cardiac actin gene that modu late its transcription in muscle cells : presence of an evolutionarily conserved repeated motif. Mol Cell Biol 1986 ; 6 : 2125-36 20. Jaynes JB, Johnson JE, Buskin JE, Gartside JN, Hauschka SD. The muscle creatine kinase gene is regulated by multiple upstream elements, including a musclespecific enhancer. Mol Cell Biol 1988; 8 62-72

21. Libri D, Mouly V, Lemonnier $\mathbf{M}$ Fiszman MY. A non muscle tropomyosin is encoded by the smooth/skeletal $\beta$ tropomyosin gene and its RNA is transcribed from an internal promoter. $J$ Biol Chem 1990 ; 265 : 3471-3

22. Heeley DA, Moir AJG, Perry SV Phosphorylation of tropomyosin during development in mammalian striated muscle. FEBS Letters 1982 ; 146 : 115-8.

23. Matsuda R, Spector D, Strohman RC. Denervated skeletal muscle displays discoordinate regulation for the synthesis of several myofibrilar proteins. Proc Natl Acad Sci USA 1984 ; 81: 1122-5.

24. Meinnel T, Libri D, Mouly V, Gros $\mathrm{D}$, Fiszman MY, Lemonnier M. Tissue specific transcriptional control of $\alpha$ and $\beta$ tropomyosins in chicken muscle development. Dev Biol 1989 ; 131 : 430-8.

25. Takeda S, Nomura Y. Presence of the tropomyosin $\beta$ chain in the dystrophic chicken breast muscle. Biomed Res $1980 ; 1$ : 176-9.

26. Izumo S, Nadal-Ginard B, Mahdavi V. Protooncogene induction and reprogram ming of cardiac gene expression produced by pressure overload. Proc Natl Acad Sci USA $1988 ; 85: 339-43$

27. Leavitt J, Latter G, Lutomski L, Goldstein D, Burbeck S. Tropomyosin isoform switching in tumorigenic human fibroblasts. Mol Cell Biol 1986; 6 : 2721-6.

28. Martin-Zanca D, Hughes SH, Barbacid $M$. A human oncogene formed by the fusion of truncated tropomyosin and tyrosine kinase sequences. Nature 1986 ; 319 : 743-8.

29. Phillips Jr GN, Fillers JP, Cohen C. Tropomyosin crystal structure and muscle regulation. J Mol Biol 1986 ; 192 : 111-31. 30. Smillie LB. Structure and function of tropomyosins from muscle and non-muscle région $\mathrm{N}$-terminale des tropomyosines musculaires, dans laquelle 8 acides aminés sur 9 sont retrouvés chez la drosophile et l'homme, alors que la région C-terminale de la protéine est très variable (d'où des aptitudes différentes à polymériser de ces isoformes).

En ce qui concerne les régions promotrices, les gènes de tropomyosines ne possèdent pas les éléments habituels des gènes s'exprimant dans les tissus différenciés, à savoir une "boîte TATA " et une "boîte CCAAT ». En revanche, ces régions présentent les séquences consensus, liant des activateurs de la transcription, retrouvées dans la plupart des gènes s'exprimant dans les tissus musculaires (séquences "CCArGG " [19], MyoD1 [20], etc.).

\section{Régulation de l'expression des tropomyosines au cours de la différenciation et du développement musculaires}

\section{- Différenciation musculaire}

La reproduction in vitro de la différenciation musculaire a été réalisée avec des cultures primaires de myoblastes ou avec des lignées myogéniques. Cela a permis d'analyser les niveaux de régulation de l'expression des gènes du programme myogénique et en particulier celle des deux gènes codant pour les isoformes $\alpha \mathrm{f}$ et $\beta$ de la tropomyosine.

A partir du gène $\beta$-tropomyosine, trois transcrits matures sont obtenus chez les oiseaux [21], auxquels correspondent trois protéines : $\mathrm{nm} \beta \mathrm{TM}$ (tissus non musculaires), $\beta$ TM (muscles striés) et $\operatorname{sm} \beta \mathrm{TM}$ (muscles lisses) (figure 4). Pendant la différenciation, l'initiation de la transcription du gène $\beta$-tropomyosine est reportée du promoteur interne (site 1') vers le promoteur distal (site 1) (figure 4), alors que la transcription du gène $\alpha f$ reste initiée à partir du promoteur distal dans les myoblastes comme dans les myotubes. La différenciation entraîne une stimulation de la transcription des deux gènes $\alpha \mathrm{f}$ et $\beta$ vraisemblablement sous l'influence de facteurs se fixant sur des séquences régulatrices des régions promotrices.
Enfin, la différenciation entraîne une modification de l'épissage des transcrits primaires des deux gènes $\alpha$ f et $\beta$ avec production des isoformes spécifiques du tissu différencié au détriment des isoformes non musculaires. Il est intéressant de noter que lors de leur différenciation, les cultures primaires de cellules myogéniques d'oiseaux produisent un phénotype différencié analogue à celui d'un muscle rapide ( $\alpha \mathrm{f}$ et $\beta$ ), et cela indépendamment du devenir ultérieur du muscle à partir duquel la culture a été obtenue. L'expression de l'isoforme $\alpha$ s peut cependant être obtenue in vitro en sous-cultivant les cellules, c'est-à-dire en les forçant à effectuer un certain nombre de divisions avant la différenciation, ce qui provoquerait une dilution progressive de facteur(s) de régulation. Ce système pourrait donc constituer un modèle intéressant pour analyser les facteurs impliqués dans la régulation du gène $\alpha$ s en conditions aneurales. - Expression des tropomyosines au cours de la maturation

La maturation du tissu musculaire consiste en l'acquisition d'un phénotype adulte et ce processus s'accompagne d'une série de modifications de l'expression des isoformes des protéines contractiles, synthétisées lors de la différenciation du tissu chez l'embryon. Par rapport aux autres protéines contractiles, les tropomyosines suivent une évolution un peu différente. Il n'y a pas à proprement parler d'isoforme embryonnaire des tropomyosines, contrairement à ce qui est observé pour la myosine par exemple. Seuls les rapports dans lesquels sont exprimées les isoformes $\alpha$ et $\beta$ varient au cours de la maturation du tissu. Chez les oiseaux, les muscles embryonnaires expriment préférentiellement l'isoforme $\beta$ tropomyosine $(\alpha f / \beta: 3 / 7$ contre $1 / 1$ dans les muscles rapides de l'adulte). Dans un muscle ultérieurement lent $\mathrm{du}$ poulet, l'anterior latissimus dorsi (ALD), l'isoforme $\alpha$ s est déjà présente à 11 jours in ovo et se substitue totalement à l'isoforme $\alpha \mathrm{f}$ avant l'éclosion. Au cours de la maturation musculaire, une diminution de la phosphorylation des isoformes $\alpha$ et $\beta$ est également observée chez les oiseaux [18] comme chez les mammifères [22]. Cette phosphorylation con- 


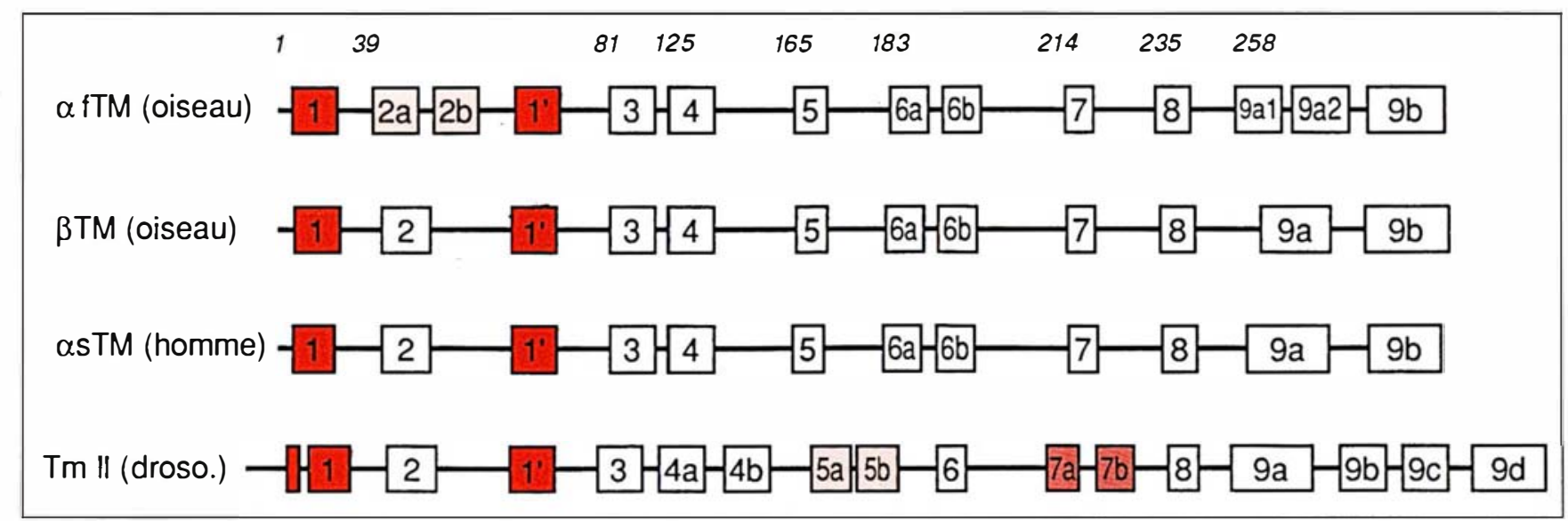

Figure 5. Représentation schématique de l'organisation exons-introns des gènes $\alpha$ fTM et $\beta$ TM des oiseaux, $\alpha$ sTM de I'homme et TmII de D. melanogaster. Les numéros au-dessus des exons indiquent les acides aminés codés par chaque exon. Les numéros à l'intérieur des exons indiquent le numéro de l'exon, affecté d'une lettre pour les exons alternatifs.

cerne l'extrémité-C terminale de la molécule et pourrait être impliquée, entre autres, dans la polymérisation des tropomyosines.

Des modifications du phénotype d'un muscle, observées au cours de la maturation in vivo, peuvent être imposées par des expériences d'innervation croisée ou de stimulation électrique. Des muscles rapides ou lents de mammiferes ou d'oiseaux peuvent être modifiés et présentent des caractéristiques biologiques, physiologiques et ultrastructurales différentes de celles de la fibre non traitée. Cela suggère que des facteurs trophiques libérés par un motoneurone lent ou rapide ainsi que des facteurs dont la production est liée à l'activité électrique exercent une action régulatrice sur l'expression ou la répression des gènes codant pour les protéines contractiles. L'ensemble des protéines contractiles est concerné par ces processus de maturation normaux ou imposés. Il est donc tentant de rechercher des blocs de régulation conduisant à une expression coordonnée des isoformes des protéines sarcomériques s'exprimant au cours de la maturation. La comparaison de la cinétique de modification des tropomyosines avec celle des chaînes légères ou des chaînes lourdes de la myosine paraît exclure l'existence d'un tel bloc de régulation. En revanche, la concertation de l'expression des tropomyosines et des troponines
$\mathrm{T}$ [23] est compatible avcc l'existence de régulations communes à ces deux groupes de protéines qui participent toutes deux au filament fin.

$\mathrm{Au}$ cours de la maturation des muscles, ou en culture de cellules myogéniques, l'expression de la $\beta$ tropomyosine semble plus modulable que celle des $\alpha$-tropomyosines. Ce phénomène pourrait signer l'existence déjà invoquée de propriétés fonctionnelles différentes de ces deux sousunités. Alors que l'on ne connaît pas de muscle n'exprimant pas d'isoforme $\alpha$-tropomyosine, l'expression de la $\beta$-tropomyosine peut être abolie sans pour autant perturber la fonction du filament fin. C'est le cas du muscle pectoral des oiseaux (muscle entièrement rapide) dont la maturation normale entraîne l'extinction de la $\beta$-tropomyosine, sous l'effet d'une régulation transcriptionnelle [24]. Après dénervation du muscle normal [23] ou dans le muscle pectoral de poulets dystrophiques [25], dans lequel l'innervation est fonctionnelle, la $\beta$-tropomyosine reste exprimée. Cela suggère que le contrôle transcriptionnel exercé relève à la fois de facteurs liés à l'innervation et de facteurs intrinsèques au muscle pectoral lui-même. Un dernier élément intéressant de ce modèle animal réside dans l'existence d'une concertation de l'expression des gènes $\alpha \mathrm{f}$ et $\beta$, qui se dégage de l'étude comparative des protéines et des mes- sagers $\alpha \mathrm{f}$ et $\beta$ qui sont exprimés dans le muscle normal et dénervé.

Un autre exemple de modulation de l'expression de la $\beta$-tropomyosine est associé à un processus pathologique, mais cette fois-ci chez les mammiferes: cette isoforme, normalement absente du muscle cardiaque de rat, est en effet retrouvée dans le muscle hypertrophié expérimentalement sous l'effet d'une sténose aortique, en même temps que sont réexprimées d'autres protéines sarcomériques présentes dans le cœur fotal [26]. La signification physiologique de ces modifications n'est pas connue, mais à la réexpression d'un programme embryonnaire pourraient être associées des différences fonctionnelles des myofibres néoformées, lors de l'augmentation de la masse musculaire du myocarde.

\section{Tropomyosines non musculaires et transformation cellulaire. Un nouvel oncogène trk associé à la tropomyosine}

La transformation cellulaire par des virus, par des oncogènes ou par des agents chimiques tératogènes entraîne des changements dans la morphologie cellulaire. L'analyse des tropomyosines synthétisées dans ces cel- 
lules transformées montre une diminution nette des isoformes ayant le plus d'affinité pour l'actine [27]. Dans la mesure où les tropomyosines non musculaires ne forment que des homodimères, cette baisse des tropomyosines à haute affinité pourrait expliquer les modifications importante, de l'ensemble des microfilaments qui participent au cytosquelette. Il est cependant difficile d'établir une relation de causalité entre les deux phénomènes, dans la mesure où des changements ont également été observés pour différentes isoformes de l'actine dans ces mêmes processus. L'existence d'un nouvel oncogène humain (trk) a été révélée par transfection d'ADN d'une tumeur du côlon humain dans des cellules NIH-3T3 [28]. Cet oncogène pro-

\section{Conclusions. Perspectives}

La diversité des isoformes des tropomyosines a longtemps constitué un frein pour étudier la fonction de ces protéines. Aujourd'hui, l'analyse des gènes fait clairement ressortir les parentés existant entre les isoformes musculaires et non musculaires, et focalise l'attention sur les régions clés de la protéine, potentiellement déterminantes sur le plan fonctionnel: polymérisation, interaction avec l'actine et avec le complexe des troponines. Ces régions peuvent maintenant être étudiées grâce aux techniques de recombinaison génétique : fabrication de tropomyosines mutées localement ou chimériques et utilisation de ces constructions dans des tests fonctionnels in vitro ou dans des

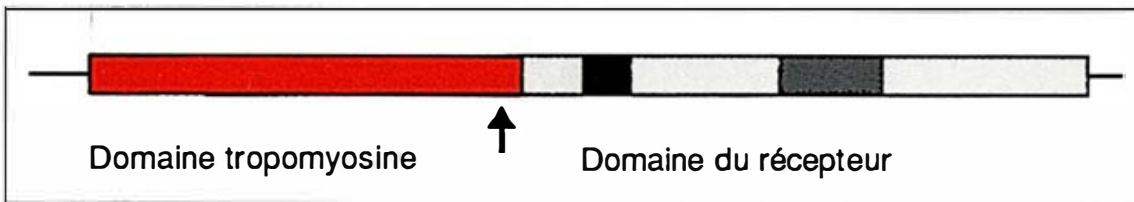

Figure 6. Schéma de I'ARNm hybride transcrit à partir de l'oncogène humain trk, formé par la recombinaison d'un gène tronqué de tropomyosine avec des séquences tyrosine kinase [28]. Les traits pleins indiquent les séquences non traduites, la boîte rouge représente les séquences dérivées du gène de tropomyosine (acides aminés 1 à 221), la boîte hachurée représente le domaine dérivé du gène du récepteur membranaire, dans lequel sont indiqués le domaine transmembranaire (boîte noire) et la séquence ayant une activité tyrosine kinase (boîte quadrillée).

vient de la recombinaison d'un gène codant pour la tropomyosine non musculaire TM30nm et la sous-unité $\alpha$ de muscle lent avec un gène codant pour un récepteur transmembranaire et son produit est une protéine hybride associant 220 aminoacides $\mathrm{N}$ terminaux de tropomyosine à une séquence codant pour une tyrosine kinase (figure 6). Le rôle de la séquence tropomyosine dans cet oncogène pourrait être de perturber la localisation du récepteur, ou de favoriser la phosphorylation de protéines du cytosquelette interagissant avec le domaine tropomyosine, ou enfin d'altérer la conformation du domaine tyrosine kinase, facilitant ainsi la phosphorylation des résidus tyrosine du site catalytique et par là l'autoactivation de cette kinase. expériences de transfection de cellules en culture. De même, l'utilisation de sondes nucléotidiques anti-sens, en arrêtant sélectivement la synthèse d'une isoforme, révélera les capacités d'une cellule à réagir lors de la disparition d'un constituant du sarcomère ou du cytosquelette (réorganisation et modification des propriétés morphologiques et fonctionnelles). Par ailleurs, les tropomyosines constituent un système exemplaire pour étudier, d'une part aussi bien le contrôle génétique associé à la différenciation du muscle (choix de promoteur, contrôle de l'épissage) que, d'autre part, la maturation de ce tissu (analyse des régulations hormonales ou neuronales sur l'expression des gènes), chez l'animal comme chez l'homme

\section{Summary}

Tropomyosins

Tropomyosins are a family of protcins, highly related throughout species, which are found in all tissucs always in association with polymerized actin ( $F$ actin). In striated muscle, tropomyosin interacts with the troponin complex and mediates the effect of calcium upon muscle contraction. In smooth muscle and non-muscle tissues, tropomyosins stabilize the actin filaments and are involved in cell motility. The diversity of tropomyosins is relevant to the existence of more than one gene and also, inside a single gene, to mRNA splicing of $5^{\prime}$ or $3^{\prime}$ terminals and of internal exons. Malignant transformation has a differential effect on the expression of the non-muscle isoforms of tropomyosin to which correspond changes into the cell cytoskeleton. Striated muscle specific isoforms of tropomyosin start to be synthesized when differentiation occurs. The expression of all muscle specific isoforms undergo changes in relation with various factors, i.e. neural input as muscle maturation proceeds, and also under some pathological conditions.
TIRÉS A PART

M. Lemonnier. 